Original article

\title{
Comparison of $\gamma$-aminobutyric acid accumulation capability in different mung bean (Vigna radiata L.) varieties under heat and relative humidity treatment, and its correlation with endogenous amino acids and polyamines
}

\author{
Yuling Ma, ${ }^{1,2}$ (D) Litao Tong, ${ }^{1}$ (D) Juan Li,${ }^{1}$ Jawad Ashraf, ${ }^{1}$ Shanshan Wang, ${ }^{1}$ Bo Zhao, ${ }^{1}$ Liya Liu, ${ }^{1}$ \\ Christophe Blecker ${ }^{2} *$ \& Sumei Zhou ${ }^{1 *}$ \\ 1 Institute of Food Science and Technology, Chinese Academy of Agricultural Sciences, No. 2 Yuan Ming Yuan West Road, Haidian \\ District, Beijing 100193, China \\ 2 Department of Food Science and Formulation, Gembloux Agro-Bio Tech, Université de Liège, Passage des Déportés 2, Gembloux, Belgium
}

(Received 4 May 2020; Accepted in revised form 8 August 2020)

Summary In this study, the accumulation of GABA and its inherent factors across different varieties of mung bean (Vigna radiata $\mathrm{L}$.) in response to heat and relative humidity (HRH) were investigated. Results showed the average GABA content in mung bean varieties was increased 7.52 times following HRH treatment, and the black mung bean variety (A8) exhibited the highest GABA accumulation capability (1.76-84.57 mg per $100 \mathrm{~g} \mathrm{DW})$. From the perspective of GABA shunt metabolites, the free glutamic acid content of mung beans significantly decreased $(P<0.05)$ after $\mathrm{HRH}$ treatment and presented a significant correlation $(P<0.05)$ with GABA content. In polyamine degradation pathway, although the average levels of spermine and spermidine of mung bean varieties significantly decreased $(P<0.05)$ after HRH treatment, no significant correlation with GABA content was identified. Hence, the GABA accumulation was predominantly attributed to GABA shunt. Besides, free amino acids including glutamic acid, serine, ornithine, arginine and glycine in mung beans showed a significant positive correlation $(P<0.05)$ with GABA content and increment following HRH treatment, which suggested that mung beans enriched in these free amino acids might accumulate higher amounts of GABA after HRH treatment and be useful for industrial applications.

Keywords Amino acids, heat and relative humidity treatment, mung bean, polyamines, $\gamma$-aminobutyric acid.

\begin{abstract}
Introduction
$\gamma$-Aminobutyric acid (GABA), a non-proteinogenic amino acid, has been extensively researched due to its health benefits including the reduction of hypertension (Nishimura et al., 2016), regulation of blood glucose (Chen et al., 2016), modulation of blood cholesterol levels (Roohinejad et al., 2009), improvement of anxiety and depression (Chuang et al., 2011) and enhancement of memory and immunity (Diana et al., 2014; Huang et al., 2019). Unfortunately, the GABA content of the brain reduces with age (Leventhal et al., 2003) and some chronic diseases (Bhagwagar et al., 2007), eventually resulting in some neurological degeneration and related disorders. However, exogenous GABA
\end{abstract}

*Correspondent: E-mails: christophe.blecker@ulg.ac.be (CB) and zhousumei@caas.cn (SZ) intake can mitigate these outcomes (Leventhal et al., 2003; Chuang et al., 2011; Diana et al., 2014). Thus, GABA-enriched food and dietary supplements have gained attention.

GABA content in soaked, germinated and fermented cereals exposed to various treatments such as heat shock (Morrison et al., 2013), cold shock (Liao et al., 2013), gaseous treatment (Komatsuzaki et al., 2007) and additives (Guo et al., 2012) has varying degrees of enhancement effects. Generally, GABA accumulation in cereals occurs due to the activation of the rate-limiting enzymes glutamate decarboxylase (GAD) of GABA shunt, which converts the endogenous and/or exogenous glutamic acid (Glu) to GABA (Khwanchai et al., 2014; Zhao et al., 2017). Yang et al (2013) and Xing et al (2007) emphasised that polyamine (PA) degradation pathway also plays a vital role in GABA 
accumulation in germinated fava bean and soya bean, mainly due to the activation of the key enzyme, diamine/polyamine oxidase (DAO/PAO), and consumption of PAs. In addition, proline (Pro) is a precursor in the synthesis of GABA through a non-enzymatic reaction (Signorelli et al., 2015). Moreover, the metabolism of Glu and PAs are closely linked to other amino acids (AAs), such as alanine (Ala), arginine (Arg) and ornithine (Orn) (Podlešáková et al., 2019).

However, all the methods mentioned are resourceintensive, requiring sufficient water and time (generally more than $24 \mathrm{~h}$ ) and resulting in the modification of the shape and/or flavour of cereals. This eventually impacts the cereal consumption patterns. It is well known that the mature dry seeds are metabolically limited, although some physiological and chemical changes occur $(\sim 7-14 \%$ moisture content). Fukumori et al. (2013) found that the heat and relative humidity (HRH) treatment $\left(50{ }^{\circ} \mathrm{C}\right.$ and $90 \% \mathrm{RH}$ or more for several hours) significantly promote GABA accumulation in mature rice seeds with extremely low moisture content (16-18.5\%). Besides, this method also prevents taste deterioration of rice during soaking, germination and drying, thereby reducing the cost of hydration and dehydration. Moreover, other grains and pulses treated with this method also presented different degrees of GABA enhancement (Fukumori et al., 2013).

Mung bean (Vigna radiata L.) is one of the most important and popular pulses due to its high nutritional value (high protein and dietary fibre, and low lipid contents). Mung beans have various nutraceutical ingredients and are recommended as an excellent nutritive pulse that can prevent chronic degenerative diseases (Ganesan \& Xu, 2018). Besides, mung bean also had a high potential to accumulate GABA (Fukumori et al., 2013; Tiansawang et al., 2016; Nikmaram et al., 2017; Chen et al., 2018). However, the capability in GABA accumulation depends on the cereal species and varieties (Khwanchai et al., 2014; Ding et al., 2016). China has cultivated various varieties of mung beans over the past 2000 years. However, GABA accumulation capability of different mung bean varieties following HRH treatment and its inherent factors have not been studied.

In the current study, thirty-four major mung bean varieties, planted across eight different provinces in China, were collected and treated by HRH. Our objectives were to compare the GABA accumulation capability in different varieties of mung bean following $\mathrm{HRH}$ treatment, and investigate its correlation with AAs and PAs. We anticipated that mung bean varieties with the highest GABA content can be screen out and the key factors that contribute to the GABA accumulation capability can be identified. Our findings will provide useful information for the development of GABA-enriched functional products.

\section{Materials and methods}

\section{Mung bean varieties}

Thirty-four mung bean (Vigna radiata L.) varieties coded as A1-A11, B12-B17, C18-C21, D22-D25, E26E28, F29-F31, G32-G33 and H34 were collected from Hebei (A), Jilin (B), Neimenggu (C), Hubei (D), Henan (E), Anhui (F), Shanxi (G) and Heilongjiang (H) provinces in China, respectively. The colour of A2, A8, A10 and D24 varieties was black, while that of others was green (Figure S1).

\section{HRH-treated mung beans}

The mung beans were treated by HRH according to the method of Fukumori et al. (2013) with some modifications. Briefly, $50 \mathrm{~g}$ of mung bean seeds was placed in a temperature and humidity test chamber (KW-TH49T, Dongguan KOWIN Testing Equipment Co., Ltd, Guangdong, China) and treated as follows: step 1, $70{ }^{\circ} \mathrm{C}, 95 \% \mathrm{RH}$ for $4 \mathrm{~h}$; step $2,40{ }^{\circ} \mathrm{C}, 70 \% \mathrm{RH}$ for $4 \mathrm{~h}$; and step $3,30{ }^{\circ} \mathrm{C}, 70 \% \mathrm{RH}$ for $4 \mathrm{~h}$.

All native and $\mathrm{HRH}$-treated mung bean samples were ground using a laboratory mill (CT 410 Cyclotec $^{\mathrm{TM}}$, FOSS Scino Co. Ltd, Suzhou, China) and stored at $4{ }^{\circ} \mathrm{C}$ until further analysis.

\section{Chemical reagents}

The GABA, putrescine (Put), spermine (Spm) and spermidine (Spd) standards were purchased from Sigma (03835, 51799, 55513 and 49761, respectively, Sigma-Aldrich, Darmstadt, Germany). AA and free AA (FAAs) mixture standard solution was provided by Wako (013-08391, 016-08641 and 015-14461, Wako Pure Chemical Industries, Ltd., Osaka, Japan). Dabsyl chloride was purchased from MCE (HY-101890; MedChemExpress, Monmouth Junction, NJ, USA). All other reagents and solvents used in the experiment were of analytical and/or HPLC grade.

\section{GABA content determination}

GABA content in native and HRH-treated mung beans was determined according to the method of Yang et al. (2013) with some modifications. Briefly, mung bean flour was extracted with $70 \%$ ethanol (ratio of powder and liquid, 1:10) at room temperature $\left(25-30{ }^{\circ} \mathrm{C}\right)$ with shaking for $30 \mathrm{~min}$. GABA contained in the supernatant layer was isolated by centrifuging at $11000 \mathrm{~g}$ at $25{ }^{\circ} \mathrm{C}$ for $10 \mathrm{~min}$. Then, $1 \mathrm{~mL}$ of the extraction was mixed with $0.2 \mathrm{~mL} \mathrm{NaHCO}$ ( $(0.4 \mathrm{~g}$ with $10 \mathrm{~mL}$ water), and $0.4 \mathrm{~mL}$ of dabsyl chloride $(20 \mathrm{mg}$ with $10 \mathrm{~mL}$ acetonitrile) was added and incubated at $70{ }^{\circ} \mathrm{C}$ for $20 \mathrm{~min}$. GABA concentrations were measured using 
HPLC (Agilent 1260, Agilent Technologies Inc., Palo Alto, CA, USA) with a ZORBAX SB-C18 reversedphase column $(5 \mu \mathrm{m}), 4.6 \times 250 \mathrm{~mm}$ i.d. Sodium acetate buffer solution $(50 \mathrm{~mm}, 69 \%)$ was regarded as mobile phase A, while mobile phase $\mathrm{B}$ was acetonitrile $(31 \%)$ at a flow rate of $1.0 \mathrm{~mL} \mathrm{~min}{ }^{-1}$ during the entire run, and the samples were detected at $436 \mathrm{~nm}$.

\section{AA and FAA determination}

The AAs in native and HRH-treated mung beans were measured according to Qin et al. (2014) with some modifications. Mung bean flour $(200 \mathrm{mg})$ was mixed with $10 \mathrm{~mL} 6 \mathrm{M} \mathrm{HCl}$ in a hydrolysis tube. Then, the lid was closed after filling with nitrogen for $1 \mathrm{~min}$. Next, the samples were hydrolysed at $110^{\circ} \mathrm{C}$ for $24 \mathrm{~h}$. Then, the samples were filtered by filter paper and the filter liquor was adjusted to $50 \mathrm{~mL}$ by water. Subsequently, $1 \mathrm{~mL}$ of liquor was taken and was dried for flushing nitrogen. Then, $1 \mathrm{~mL}$ of $0.02 \mathrm{M} \mathrm{HCl}$ was used for reconstitution and filtered by using a $0.45-\mu \mathrm{m}$ filter membrane. After that, the AAs were analysed using an amino acid analyser (L-8800; Hitachi, Tokyo, Japan). Additionally, the tryptophan (Trp) was analysed alone after alkaline hydrolysis (Guo et al., 2017). Specifically, $50 \mathrm{mg}$ samples were hydrolysed with $1.0 \mathrm{~mL} 10 \% \mathrm{KOH}$ for $18 \mathrm{~h}$ at $40{ }^{\circ} \mathrm{C}$. Next, $0.2 \mathrm{~mL}$ $5 \%$ dimethylaminobenzaldehyde and $0.2 \mathrm{~mL} \quad 1 \%$ sodium nitrate were added to the hydrolysed mixture. Then, the mixture was placed in an ice water bath and $5 \mathrm{~mL}$ concentrated hydrochloric acid was added. The mixture was shaken and incubated at $40^{\circ} \mathrm{C}$ for $45 \mathrm{~min}$ before the absorbance of the supernatant was measured at $590 \mathrm{~nm}$.

The FAAs from native and HRH-treated mung beans were characterised by the method of Zhao et al. (2017) with some modifications. Briefly, $0.5 \mathrm{~g}$ of mung bean flour was extracted with $0.1 \mathrm{M} \mathrm{HCl}$ (ratio of powder and liquid, 1:10) at room temperature (25$30{ }^{\circ} \mathrm{C}$ ) under sonication for $10 \mathrm{~min}$. Then, the mixture solution was centrifuged at $4193 \mathrm{~g}$ for $15 \mathrm{~min}$ at room temperature $\left(25-30^{\circ} \mathrm{C}\right)$ and the supernatant was adjusted to $5 \mathrm{~mL}$. Subsequently, $1 \mathrm{~mL}$ supernatant was mixed with $1 \mathrm{~mL}$ of $8 \%$ sulfosalicylic acid to purify the FAAs. After centrifugation at $16770 \mathrm{~g}$ for $15 \mathrm{~min}$ at room temperature $\left(25-30^{\circ} \mathrm{C}\right), 1 \mathrm{~mL}$ of supernatant was taken and was dried for flushing nitrogen. Then, $1 \mathrm{~mL}$ of $0.02 \mathrm{M} \mathrm{HCl}$ was used for reconstitution and filtered by using a $0.45-\mu \mathrm{m}$ filter membrane. Subsequently, the FAAs were analysed using the above-mentioned amino acid analyser.

\section{PA determination}

Put, Spm and Spd from native and HRH-treated mung bean were analysed using HPLC as described by
Xing et al. (2007) with slight modifications. Mung bean flour $(0.25 \mathrm{~g})$ was mixed with $2.5 \mathrm{~mL}$ of $5 \%$ $\mathrm{HClO}_{4}$ with $1 \mathrm{~h}$ in an ice bath. Then, the mixture solution was centrifuged at $8520 \mathrm{~g}$ for $20 \mathrm{~min}$ at $4{ }^{\circ} \mathrm{C}$. Subsequently, $500 \mu \mathrm{L}$ of supernatant, $1 \mathrm{~mL} 2 \mathrm{~m}$ $\mathrm{NaOH}$, and $10 \mu \mathrm{L}$ benzoyl chloride were mixed and vortexed for about $20 \mathrm{~s}$, and incubated for about $20 \mathrm{~min}$ at $37{ }^{\circ} \mathrm{C}$. Next, $2 \mathrm{~mL}$ of saturated $\mathrm{NaCl}$ was used to stop the reaction. Ether $(2 \mathrm{~mL})$ was added for extraction. After centrifuging at $1500 \mathrm{~g}$ for $5 \mathrm{~min}$, we dried the ether phase $(1 \mathrm{~mL})$ by evaporation. Then, $100 \mu \mathrm{L}$ methanol was added to redissolve it. And the measurement of PA concentration was processed by HPLC with ZORBAX SB-C18 reverse-phase column $(5 \mu \mathrm{m}), 4.6 \times 250 \mathrm{~mm}$ i.d. Phase A $(36 \%)$ was water, while phase B $(64 \%)$ was methanol at a flow rate of $0.6 \mathrm{~mL} \min ^{-1}$ during the elution time. Reading was detected at $254 \mathrm{~nm}$.

\section{Statistical analysis}

The data of the experiments were replicated three times. The statistical analysis was performed using SPSS version 11.5. Significant differences were calculated by the variance (ANOVA) test and the Duncan multiple range test. The Box-Charts were performed by OriginPro 8. The analysis of Pearson's correlation was applied to evaluate the correlation of the changes in metabolites, and the visualisation of the correlation coefficients in a heat map was performed using MultiExperiment Viewer version 4.4.0 (http://www. tm4.org/mev/).

\section{Results and discussion}

\section{GABA contents in native and HRH-treated mung beans}

The GABA contents in different native and HRH-treated mung bean varieties are presented in Fig. 1. GABA contents in native mung beans were markedly low, with significant differences $(P<0.05)$ among varieties, ranging from 0.63 to $7.16 \mathrm{mg}$ per $100 \mathrm{~g}$, similar to the $1.7 \mathrm{mg}$ per $100 \mathrm{~g}$ DW reported by Fukumori et al. (2013), but lower (13.25 mg per $100 \mathrm{~g} \mathrm{DW})$ than that reported by Tiansawang et al. (2016). These variances might be due to differences in methods of determination, and/or mung bean varieties were investigated. However, we found that the average GABA content in mung beans following HRH treatment increased from 4.13 to $31.06 \mathrm{mg}$ per $100 \mathrm{~g} \mathrm{DW}$ (about 7.52-times). Different mung bean varieties had large differences, ranging from 17.15 to $84.57 \mathrm{mg}$ per $100 \mathrm{~g}$ DW. As shown in Figure S2, there were no differences in GABA content across both native and HRH-treated mung bean varieties from provinces (A-F). Although $\mathrm{G}$ and $\mathrm{H}$ provinces presented some 

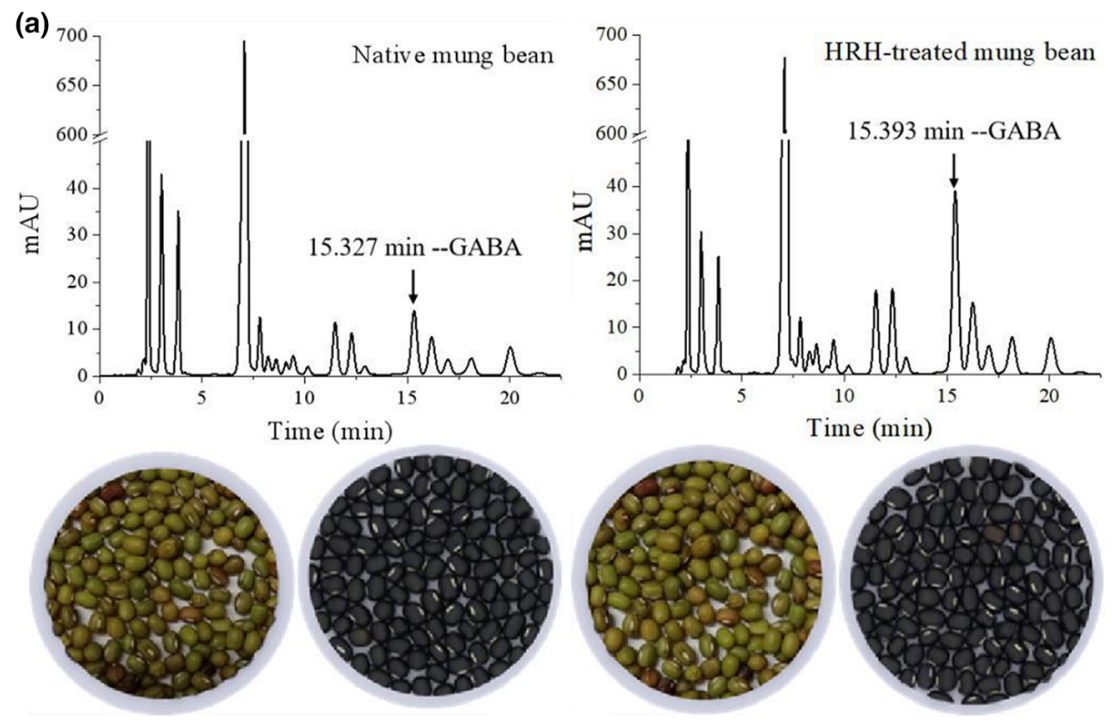

Figure 1 Representative HPLC patterns (a) and GABA contents (b) of native and $\mathrm{HRH}$ treated mung beans. [Colour figure can be viewed at wileyonlinelibrary.com]

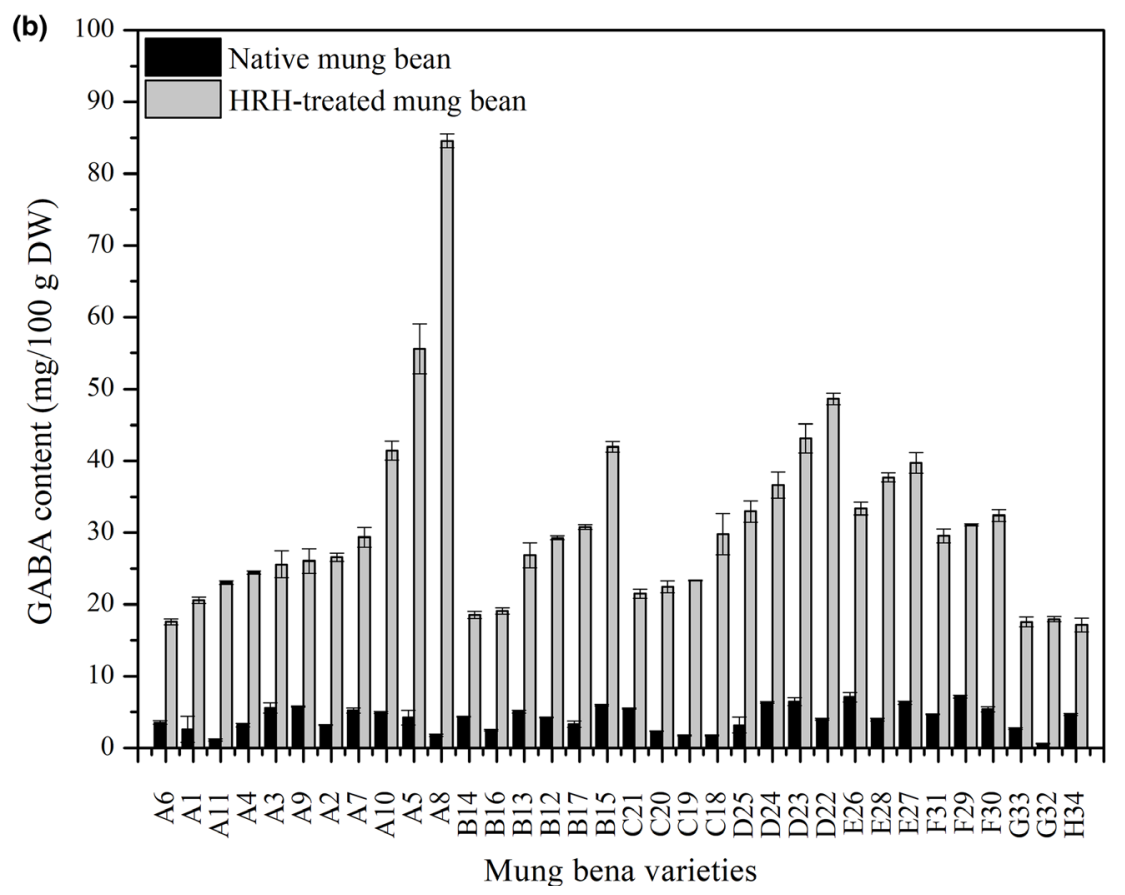

differences with other provinces, given that only one to two mung bean varieties were collected from these provinces, they might not be truly representative of the actual differences. Therefore, no distinct differences were observed in GABA content in both native and HRH-treated mung beans among the different provinces.

Of note, the black mung bean variety (A8) exhibited the highest GABA content ( $84.57 \mathrm{mg}$ per $100 \mathrm{~g} \mathrm{DW}$ ) after HRH treatment, which was equivalent to 48.05 times more concentrated than native mung bean (1.76 mg per $100 \mathrm{~g} \mathrm{DW}$ ). Tiansawang et al. (2016) reported that the highest GABA content was $80.68 \mathrm{mg}$ per $100 \mathrm{~g} \mathrm{DW}$ after $6 \mathrm{~h}$ of soaking and $24 \mathrm{~h}$ of incubation in germinated mung bean. Chen et al. (2018) showed that the optimum conditions for GABA enrichment in mung bean germination were $\mathrm{pH} 5.0$, culture temperature $30{ }^{\circ} \mathrm{C}$ and stress time $24 \mathrm{~h}$. Under these conditions, GABA content increases to $24.41 \mathrm{mg}$ per $100 \mathrm{~g} \mathrm{FW}$. Ali et al. (2015) presented that the 
GABA content in mung bean was $1.22 \mathrm{~g} \mathrm{~kg}^{-1}$ dry weight after soaking for $18 \mathrm{~h}$, boiling for $40 \mathrm{~min}$, fermenting with MARDI's Rhizopus sp. 5351 strains for $48 \mathrm{~h}$ at $30^{\circ} \mathrm{C}$ and aerobically incubating for $48 \mathrm{~h}$ at $30{ }^{\circ} \mathrm{C}$. These results indicated that HRH-treated mung bean variety (A8) also had a high GABA content compared with germinated and fermented mung bean. However, HRH treatment was more efficient than germination in terms of time and water consumption. GABA content of HRH-treated A8 mung bean variety was also higher than of some reported additive-free germinated cereals, such as germinated wheat, rice, soya bean and foxtail millet (Youn et al., 2011; Khwanchai et al., 2014; Xu \& Hu, 2014; Sharma et al., 2018). Therefore, HRH treatment was identified as a suitable method for the accumulation of GABA in mung beans, especially the black mung bean variety (A8), which has great potential for the development of natural GABA-enriched functional products.

The HRH treatment activates the metabolism regulation system and hence increases the GABA accumulation (Locy et al., 2000). The differences in GABA accumulation capability across different mung bean varieties might be due to the differences in genotypespecific types and climate conditions, resulting in the different GABA metabolic profiles (Khwanchai et al., 2014; Ding et al., 2016). Metabolism regulation of GABA relies on GABA shunt, PA degradation pathway and non-enzymatic reaction of Pro (Xing, et al., 2007; Yang et al., 2013; Signorelli et al., 2015), and some other AAs closely linked to GABA synthesis, such as Arg and Orn (Podlešáková et al., 2019). Hence, to explore the differences in the inherent factors contributing to GABA accumulation capability across different mung bean varieties, the metabolites including AAs, FAAs and PAs were also investigated.

\section{AA contents in native and $\mathrm{HRH}$-treated mung beans}

AAs play an important role in human health and are inextricably linked to GABA accumulation, such as GABA precursors Glu and Pro. Nonogaki et al. (2010) reported that early imbibition of the dry seeds re-establishes metabolism. This process is largely affected by environmental conditions. Under HRH treatment, mung beans were exposed to an environment of hypoxia and high relative humidity, resulting in low absorption of water (Fukumori et al., 2013) and might cause metabolism of AAs. To investigate the effects of HRH treatment on AAs and the correlation with GABA, Fig. 2 describes the AA contents from native and $\mathrm{HRH}$-treated mung beans. Results showed that the AA composition was similar in different mung bean varieties. The predominant AAs in mung beans were non-essential amino acids (NEAA, $11.28-15.67 \%$ DW), and the average Glu content was the highest (44.64 mg per g DW), followed by Asp (26.67 $\mathrm{mg}$ per g DW) and Arg (14.87 mg per g DW). These concentrations were supported by Shi et al (2016). The content range of total essential AAs (T-EAA) in mung beans was $7.83-10.28 \% \mathrm{DW}$; the highest content of EAAs was Leu $(18.52 \mathrm{mg}$ per $\mathrm{g}$ DW), followed by Lys (17.26 mg per g DW) and Phe (14.32 mg per g DW). However, mung beans lacked Cys, Trp and Met, with average contents of 1.29, 1.62 and $1.99 \mathrm{mg}$ per g DW, respectively. Nikmaram et al (2017) also reported that pulses were a relatively poor source of sulphur-containing amino acids.

After HRH treatment, we noticed that most of AAs were not changed. However, the average content of Ser and Tyr in mung beans significantly increased by $<10 \%$ (Ser from 10.84 to $11.61 \mathrm{mg}$ per g DW, $P<0.01$; Tyr from 5.47 to $5.86 \mathrm{mg}$ per $\mathrm{g} D W$, $P<0.05)$, whereas Val and Ile significantly decreased (Val from 13.20 to $12.46 \mathrm{mg}$ per g DW, $P<0.01$; Ile from 10.44 to $9.96 \mathrm{mg}$ per g DW, $P<0.05$ ). This indicated that HRH treatment might cause the degradation and/or synthesis of some proteins (Nonogaki et al., 2010). Yang et al. (2013) and Signorelli et al. (2015) reported that Glu and Pro are substrates for GABA synthesis. Although our results showed that the content of Glu and Pro was enriched in mung beans, their contents were not changed after $\mathrm{HRH}$ treatment. This might be because most of these AAs are locked in proteins and cannot be released. Hence, further research focused on FAA contents.

\section{FAA contents in native and HRH-treated mung beans}

Figure 3 shows the FAA profiles from native and HRH-treated mung beans, and Figure S3 shows the corresponding chromatograms. The results clearly described a large difference in FAA contents in different mung bean varieties. The free Glu (F Glu), F Arg and $F$ Asp were the most abundant FAAs in mung beans, and their contents in different varieties were widely ranged, 47.14-134.04, 45.06-121.77, and 21.55$80.360 \mathrm{mg}$ per $100 \mathrm{~g}$ DW, respectively. Ali et al. (2015) also reported a similar results, in which F Glu, $\mathrm{F}$ Arg and F Asp were higher than other FAAs in mung bean, and their contents were $0.47,0.46$ and $0.43 \mathrm{~g}$ per kg dry weight, respectively.

We also observed that HRH treatment significantly changed the FAA profiles and significantly increased $(P<0.01)$ the total FAA (T-FAA) content $(344.10$ 521.47 to $377.97-568.11 \mathrm{mg}$ per $100 \mathrm{~g} \mathrm{DW}$, respectively). Combined with the changes in AAs, we speculated that the seed components of mung bean were decomposed and/or transformed during HRH treatment, especially the mutual transformation of FAAs. Specifically, most of the FAA contents in mung beans including GABA and other FAAs (Ala, $\alpha$-ABA, $\beta$-Ala, Gly, Ser, PEA, Thr, 
Figure 2 Amino acid contents of native and HRH-treated mung beans. Cysteine (Cys), tryptophan (Trp), methionine (Met), tyrosine (Tyr), threonine (Thr), isoleucine (Ile), valine (Val), phenylalanine (Phe), lysine (Lys), leucine (Leu), histidine (His), glycine (Gly), proline (Pro), alanine (Ala), serine (Ser), arginine (Arg), aspartic acid (Asp) and glutamic acid (Glu). Essential amino acids (EAA). N means non. $\mathrm{T}$ stands for total. 'A and $\mathrm{B}$ ' and 'a and b' represent significant differences $(P<0.01$ and 0.05 , respectively $)$ between native and HRH-treated mung bean within the same column; same as follows. [Colour figure can be viewed at wileyonline library.com]

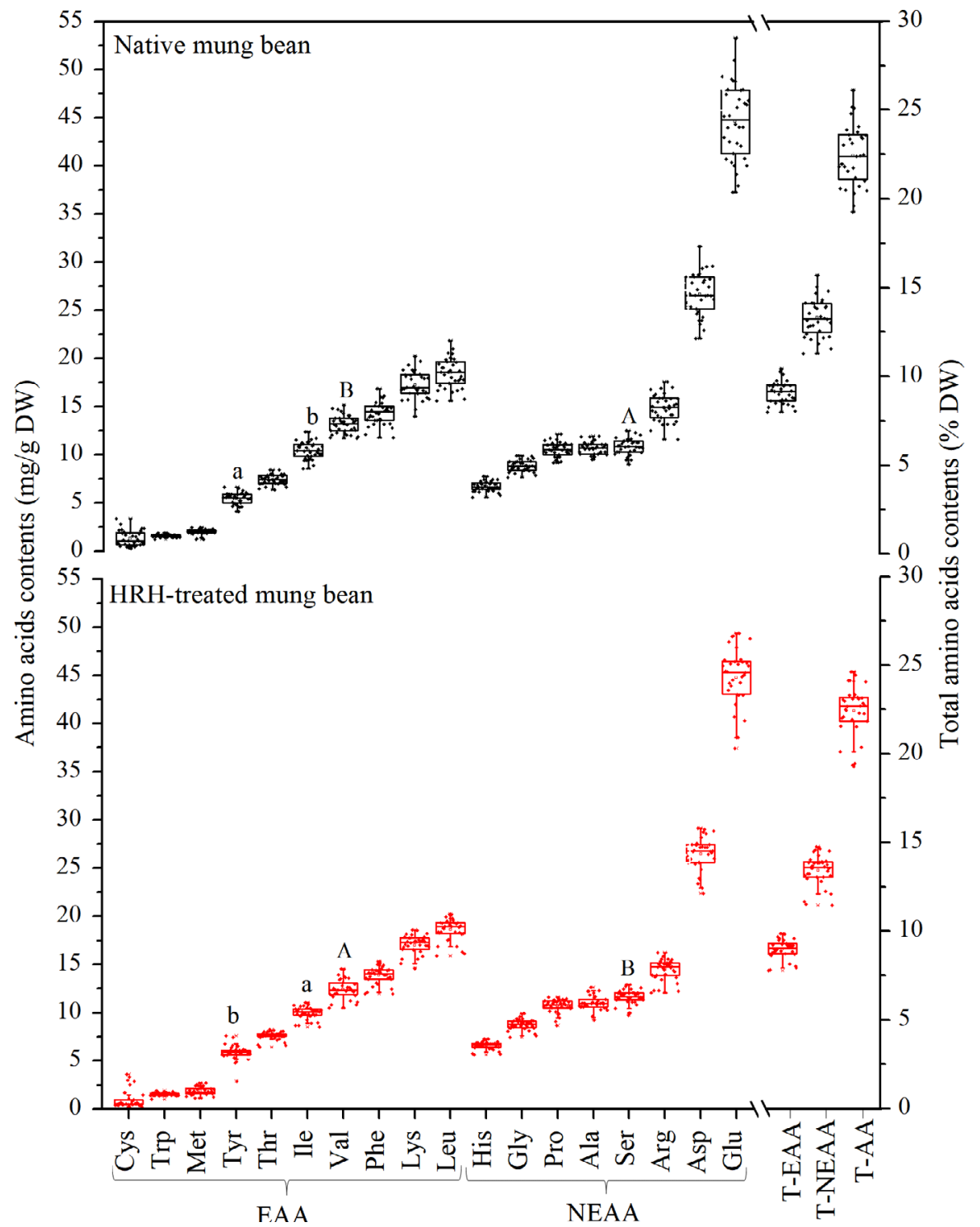

Lys, $\mathrm{EOHNH}_{2}$, Tau, Leu, Tyr and Cit) significantly increased $(P<0.01)$ after HRH treatment. Particularly, some FAAs (Tyr, EOHNH 2 , Lys, Leu, Gly and Thr) were increased more than 1.5-5 times. However, F Glu and $\mathrm{F}$ Asp contents, enriched in mung beans, were significantly decreased (77.68 to $64.14 \mathrm{mg}$ per $100 \mathrm{~g}$ DW; 55.67 to $43.82 \mathrm{mg}$ per $100 \mathrm{~g}$ DW, respectively, $P<0.01$ ). $\mathrm{Xu} \& \mathrm{Hu}(2014)$ stated that although the F Glu content of soya bean sprouts increased significantly during germination, the F Glu content of germinated soya bean at $32{ }^{\circ} \mathrm{C}$ was lower than that at 19 and $25^{\circ} \mathrm{C}$ due to the changes in the activities of GAD and GABA transaminase of GABA shunt.

Early follow-up at start of the imbibition of dry seeds re-establishes metabolism (Nonogaki et al.,
2010). Hence, the changes in FAAs might be due to the fact that mung beans under HRH treatment with a high relative humidity $(95 \% \mathrm{RH})$ absorbed some amounts of moisture and were exposed to high temperatures and low-oxygen environment (Fukumori et al., 2013). These environmental factors might have transformed various biochemical components due to enzymatic and non-enzymatic reactions. These reactions degrade and transform proteins, peptides and AAs, resulting in the changes to the FAA composition (Signorelli et al., 2015; Kim et al., 2015). Particularly, the levels of Glu, which is the precursor of GABA shunt, significantly decreased, whereas GABA content increased significantly $(P<0.05)$. This suggested that metabolic responses to $\mathrm{HRH}$ treatment might result in 


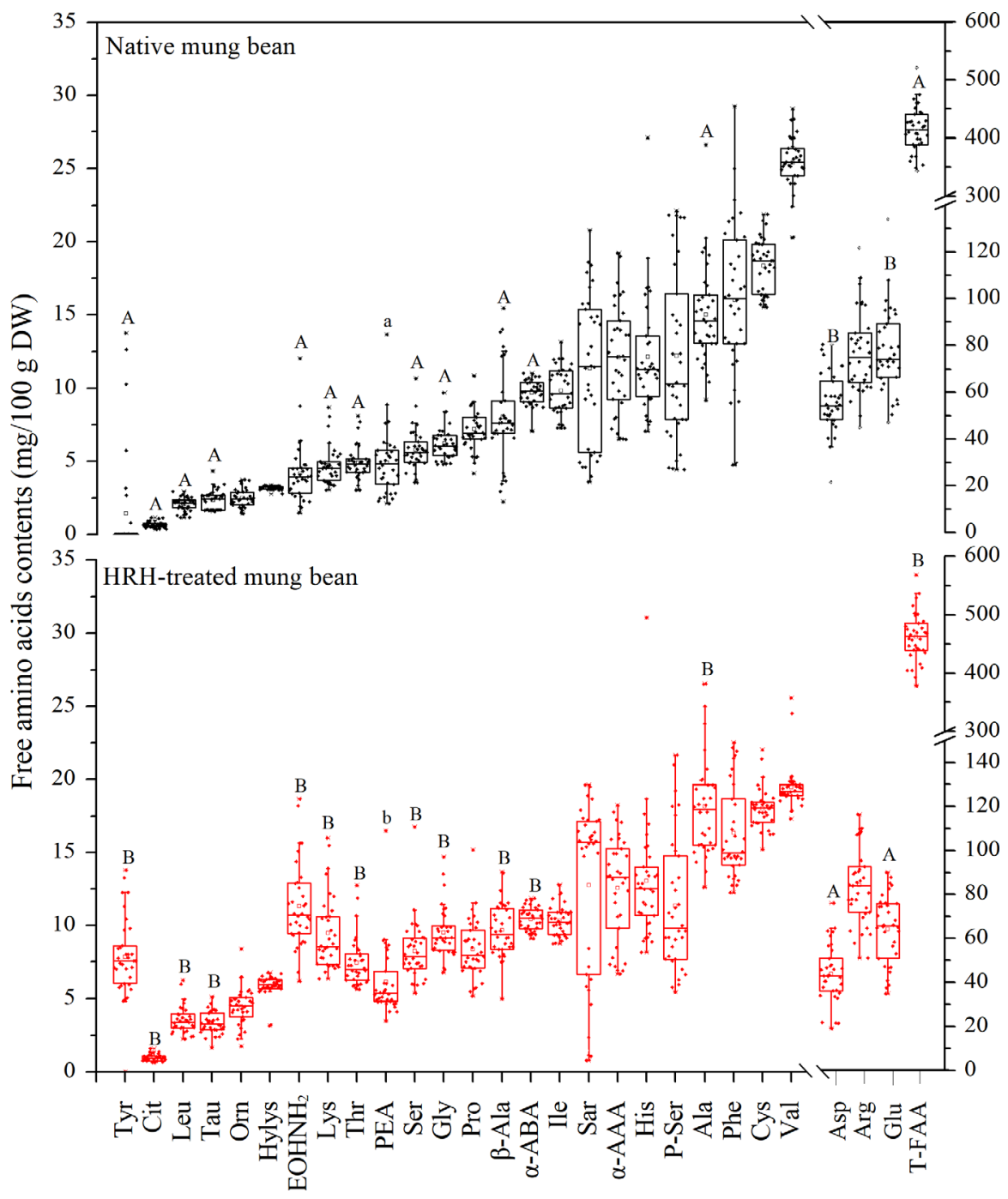

Figure 3 Free amino acid contents of native and HRH-treated mung beans. F means free. Citrulline (Cit), taurine (Tau), ornithine (Orn), hydroxylysine (Hylys), ethanolamine $\left(\mathrm{EOHNH}_{2}\right)$, phosphorylethanolamine (PEA), $\beta$-alanine ( $\beta$-Ala), $\alpha$-aminobutyric acid $(\alpha$-ABA), sarcosine (Sar), $\alpha$-aminoadipicacid $(\alpha$-AAA), phosphoserine (P-Ser). T-FAA means total free amino acid. Same as follows. [Colour figure can be viewed at wile yonlinelibrary.com] a momentary cytoplasmic acidification, activating GAD, which can convert F Glu to GABA. In addition, the differential effects of the metabolic rates caused redistribution of AA (Mayer et al., 1990). However, further studies are required to illustrate the mechanisms involved in the FAA changes in HRHtreated mung bean.

\section{PA contents in native and HRH-treated mung beans}

PAs are molecules with regulatory functions in plant abiotic stress tolerance. Put, Spm and Spd in PAs are precursors of GABA, which are catalysed by DAO and/or PAO and converted into GABA via the formation of the $\gamma$-aminobutyraldehyde intermediate (Wakte et al., 2011; Zhao et al., 2017). Figure 4 presents Put, Spm and Spd contents of native and HRH-treated mung beans. Results showed that the average contents of Put, Spd and Spm were 30.82, 493.28 and $691.81 \mathrm{nmol}$ per g DW, respectively. After HRH treatment, the average contents of Spd and Spm were lesser than that those of native mung beans, whereas no changes were observed in Put content. Yang et al. (2013) inferred that the contribution of PA degradation pathway in GABA synthesis was about $30 \%$ in the germinating fava bean under hypoxia. Thus, the decreases in Spd and Spm levels in HRH-treated mung bean might be related to GABA accumulation.

\section{Correlation between GABA and endogenous amino components including AAs, FAAs and PAs in mung beans under $\mathrm{HRH}$ treatment}

Table 1 shows the correlation between GABA including content and increment in mung beans following $\mathrm{HRH}$ treatment and endogenous amino compounds 


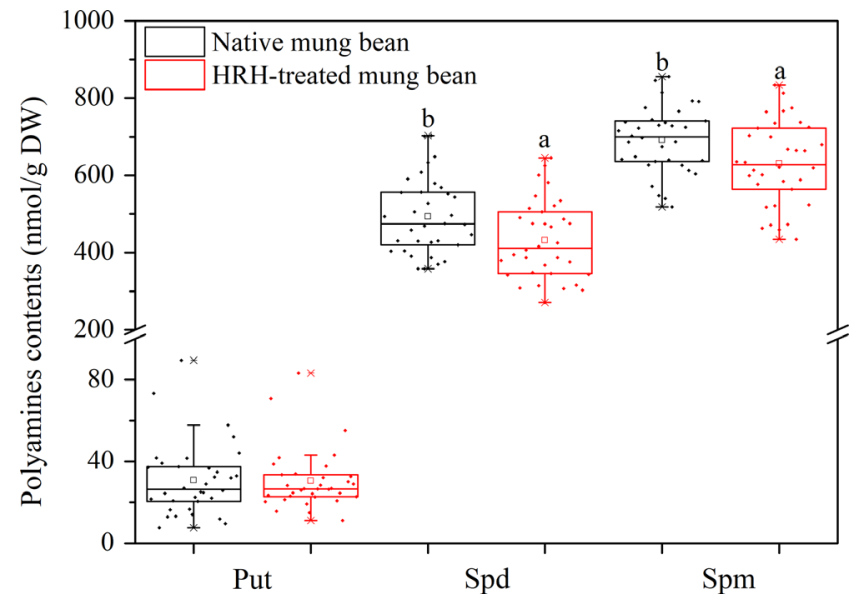

Figure 4 Putrescine (Put), spermine (Spm) and spermidine (Spd) contents of native and HRH-treated mung beans. [Colour figure can be viewed at wileyonlinelibrary.com]

including AAs, FAAs and PAs in native mung beans. Results showed that both GABA content and increment in mung beans following HRH treatment were significantly positively correlated $(P<0.05)$ with some FAAs (Glu, Ser, Orn, Arg and Gly) in native mung beans. The Glu is the precursors of GABA synthesis, which can convert Glu to GABA by activating key enzyme GAD (Khwanchai et al., 2014; Zhao et al., 2017). The Arg and Orn relate to Glu metabolism by Asp aminotransferase and Orn cycle, and also link with PA metabolism by their corresponding decarboxylase (Podlešáková et al., 2019). However, the further studies are required to give a precise explanation for their relations. Although Pro and PAs are precursors of GABA synthesis (Xing et al., 2007; Yang et al., 2013; Signorelli et al., 2015), no significant correlations were observed. The AAs, which are mainly derived from proteins, also presented no significant correlation with GABA. Tiansawang et al. (2016) suggested that legumes had a higher protein content than sesame, resulting germinated legumes produce higher GABA than sesame. Roohinejad et al. (2011) reported that a significant positive correlation $(P<0.05)$ was observed between protein, Glu and GABA contents before and after pre-germinated brown rice. However, $\mathrm{Xu} \& \mathrm{Hu}$ (2014) found that the correlation between

Table 1 Correlation between GABA including content and increment in mung beans following HRH treatment and endogenous amino compounds including AAs, FAAs and PAs in native mung beans

\begin{tabular}{|c|c|c|c|c|c|}
\hline AA content & GABA content & GABA increment & FAA content & GABA content & GABA increment \\
\hline Cys & -0.054 & -0.049 & $\mathrm{EOHNH}_{2}$ & -0.056 & -0.11 \\
\hline Trp & -0.013 & -0.048 & Lys & 0.267 & 0.24 \\
\hline Met & -0.305 & -0.313 & Thr & 0.293 & 0.25 \\
\hline Tyr & 0.263 & 0.207 & PEA & 0.027 & 0.01 \\
\hline Thr & 0.001 & -0.027 & Ser & $0.466^{\dagger}$ & $0.502^{\dagger}$ \\
\hline Ile & 0.099 & 0.072 & Gly & $0.390^{*}$ & $0.366^{\ddagger}$ \\
\hline Val & 0.13 & 0.102 & Pro & -0.129 & -0.127 \\
\hline Phe & 0.19 & 0.153 & $\beta$-Ala & 0.143 & 0.09 \\
\hline Lys & 0.17 & 0.138 & $\alpha-A B A$ & 0.156 & 0.08 \\
\hline Leu & 0.098 & 0.070 & Ile & 0.194 & 0.11 \\
\hline His & 0.096 & 0.060 & Sar & -0.102 & -0.07 \\
\hline Gly & 0.12 & 0.090 & $\alpha-A A A$ & -0.067 & -0.07 \\
\hline Pro & 0.033 & 0.001 & His & -0.207 & -0.212 \\
\hline Ala & 0.04 & 0.012 & P-Ser & 0.205 & 0.19 \\
\hline Ser & -0.002 & -0.021 & Ala & 0.284 & 0.23 \\
\hline Arg & 0.133 & 0.101 & Phe & -0.258 & -0.32 \\
\hline Asp & 0.057 & 0.035 & Cys & -0.012 & -0.03 \\
\hline Glu & 0.095 & 0.067 & Val & 0.323 & 0.25 \\
\hline FAA content & - & - & Asp & -0.334 & -0.29 \\
\hline GABA & 0.132 & 0.001 & Arg & $0.373^{\ddagger}$ & $0.375^{\ddagger}$ \\
\hline Cit & 0.107 & 0.02 & Glu & $0.485^{\dagger}$ & $0.482^{\dagger}$ \\
\hline Tyr & -0.315 & -0.32 & & & \\
\hline Leu & 0.306 & 0.23 & PA content & - & - \\
\hline Tau & -0.202 & -0.17 & Put & 0.28 & 0.33 \\
\hline Orn & $0.446^{\dagger}$ & $0.452^{\dagger}$ & Spd & 0.147 & 0.18 \\
\hline Hylys & 0.118 & 0.07 & Spm & 0.121 & 0.08 \\
\hline
\end{tabular}

AAs, amino acids; FAAs, free amino acids; $\mathrm{HRH}$, heat and relative humidity.

${ }^{\dagger}$ Correlation is significant at the 0.01 level.

${ }^{\ddagger}$ Correlation is significant at the 0.05 level (two-tailed). 
GABA and Glu in soya beans during germination declined gradually with the increase in germination temperature. Morrison et al. (2013) presented that the total protein in seeds may not always correlate with GABA and that the best method to screen high GABA concentration in a soy product is to select the cultivar with high seed concentrations of Glu and/or GABA. Therefore, the correlation between GABA and related components differs across various crop types and processing methods. Our results indicated that the mung bean seeds enriched in these FAAs (Glu, Ser, Orn, Arg and Gly) tended to show a better potential to produce GABA under HRH treatment. This provided a useful method to screen for the GABAenriched mung bean varieties.

The metabolites that showed a significant difference after HRH treatment include four types of AAs, sixteen kinds of FAAs and two kinds of PAs. The Pearson correlation analysis and hierarchical clustering analysis (HCA) of the accessions were performed to illustrate their correlations (Fig. 5). From the perspective of changes in GABA, a significantly negative correlation between the changes of GABA and F Glu was observed $(r=-0.697, P<0.01)$. Collectively, the changes in Glu, F Glu, F Pro and PAs in mung bean under HRH treatment and their correlation with GABA confirmed that the accumulation of GABA can be mainly attributed to the consumption of endogenous F Glu in GABA shunt. Contrarily, a significantly positive correlation was observed between the changes in GABA and some FAAs (Thr, Ser, Leu, Gly, Lys, Tyr, $\mathrm{EOHNH}_{2}$ and Ala: $r=0.817,0.813$, $0.780,0.775,0.736,0.633,0.604$ and 0.526 , respectively, $P<0.01)$. This suggested that the changes in these FAAs were synchronised with GABA in mung beans under HRH treatment, and HCA findings supported this. Kim et al. (2015) and Komatsuzaki et al. (2007) also found that the levels of most of FAAs increased with the accumulation of GABA in a lowoxygen environment. These might be relate to the inhibition of respiration and the block of adenosine triphosphate, which caused the relative contents of the FAAs that participate in the tricarboxylic acid cycle to increase (Chen et al., 2019b). Previous researches have

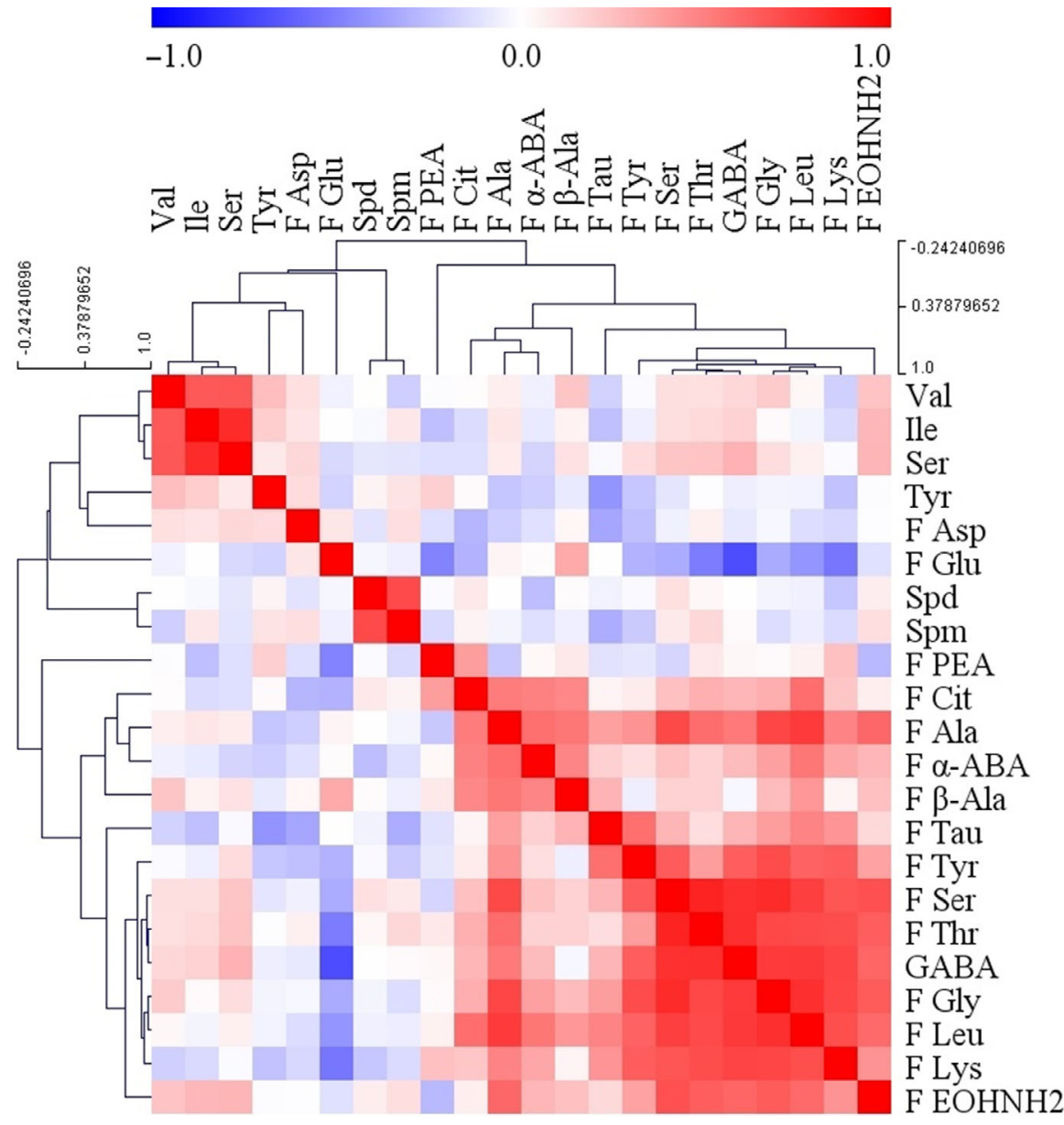

Figure 5 Correlation matrix of metabolite changes of thirty-four mung bean varieties under HRH treatments. Each square indicates Pearson's correlation coefficient for a pair of compound changes, and the value of the correlation coefficient is represented by the intensity of blue or red colour, as indicated on the colour scale. [Colour figure can be viewed at wileyonlinelibrary.com] 
confirmed that energy metabolism plays a key role in changes of nutritional and quality properties including FAAs of mung bean sprouts (Chen et al., 2018a, Chen et al., 2018b; Chen et al., 2019a, Chen et al., 2019b). However, the dynamic changes, metabolomics and energy status in mung bean seeds under HRH treatment have not been studied. Therefore, further researches about these aspects might be useful to illuminate their relationship.

\section{Conclusion}

HRH treatment was highly efficient in promoting GABA accumulation in mung beans. The average level of GABA in different mung bean varieties was increased by more than 7.5 times after processing (4.13-31.06 mg per $100 \mathrm{~g}$ DW). Specifically, the black mung bean variety (A8) exhibited the highest GABA accumulation (48 times; $1.76-84.57 \mathrm{mg}$ per $100 \mathrm{~g} \mathrm{DW}$ ) after $\mathrm{HRH}$ treatment. Investigation of the changes and correlations of metabolites in mung bean varieties under $\mathrm{HRH}$ treatment revealed that the difference in GABA accumulation capability in varieties was mainly attributed to endogenous $F$ Glu in GABA shunt. Mung bean varieties enriched in endogenous F Glu, F Ser, F Orn, F Arg and/or F Gly tended to have a better potential to accumulate GABA. Our results can aid to screen GABA-enriched mung bean varieties and yield useful products.

\section{Acknowledgments}

This study was funded by the 'Special Fund for Modern Agricultural Industrial Technology System' (CARS-08-G19) and 'Central Public-interest Scientific Institution Basal Research Fund' (No. Y2020PT33).

\section{Conflict of interest}

The authors declared that there is no conflict of interest in this work.

\section{Author contribution}

Yuling Ma: Conceptualization (lead); Data curation (lead); Formal analysis (lead); Investigation (lead); Methodology (equal); Project administration (lead); Resources (lead); Software (lead); Supervision (equal); Validation (lead); Visualization (lead); Writing-original draft (lead); Writing-review \& editing (lead). Litao Tong: Conceptualization (equal); Data curation (equal); Formal analysis (equal); Funding acquisition (equal); Investigation (equal); Methodology (equal); Project administration (equal); Supervision (equal); Writing-original draft (equal); Writing-review \& editing (equal). Juan Li: Data curation (equal); Formal analysis (supporting); Funding acquisition (supporting); Methodology (lead); Software (supporting). Jawad Ashraf: Data curation (supporting); Formal analysis (equal); Methodology (supporting); Software (equal); Visualization (supporting); Writing-original draft (supporting); Writing-review \& editing (supporting). Shanshan Wang: Methodology (supporting); Software (supporting); Visualization (supporting); Writing-original draft (supporting); Writing-review \& editing (supporting). Bo Zhao: Data curation (supporting); Formal analysis (equal); Investigation (supporting); Software (supporting); Writing-original draft (supporting). Liya Liu: Conceptualization (supporting); Investigation (equal); Validation (supporting); Writingreview \& editing (supporting). Christophe Blecker: Conceptualization (supporting); Data curation (supporting); Formal analysis (supporting); Funding acquisition (supporting); Project administration (lead); Supervision (lead); Writing-original draft (supporting). Sumei Zhou: Conceptualization (lead); Data curation (equal); Formal analysis (supporting); Funding acquisition (lead); Investigation (equal); Methodology (supporting); Project administration (lead); Resources (supporting); Software (supporting); Supervision (lead); Validation (lead).

\section{Ethical approval}

Ethics approval was not required for this research.

\section{Peer Review}

The peer review history for this article is available at https://publons.com/publon/10.1111/ijfs.14771.

\section{Data availability statement}

The data that support the findings of this study are available from the corresponding author upon reasonable request.

\section{References}

Ali, N.M., Yeap, S.-K., Yusof, H.M. et al. (2015). Comparison of free amino acids, antioxidants, soluble phenolic acids, cytotoxicity and immunomodulation of fermented mung bean and soybean. Journal of the Science of Food and Agriculture, 96, 1648-1658.

Bhagwagar, Z., Wylezinska, M., Jezzard, P. et al. (2007). Reduction in occipital cortex gamma-aminobutyric acid concentrations in medication-free recovered unipolar depressed and bipolar subjects. Biological Psychiatry, 61, 806-812.

Chen, L., Zhao, H.Z., Zhang, C., Lu, Y.J., Zhu, X.Y. \& Lu, Z.X. (2016). $\gamma$-Aminobutyric acid-rich yogurt fermented by Streptococcus salivarius subsp. thermophiles fmb5 apprars to have anti-diabetic effect on streptozotocin-induced diabetic mice. Journal of Functional Foods, 20, 267-275.

Chen, J., Wu, J.H., Lin, K.Y., Si, H.P., Gu, Z.X. \& Li, Y. (2018). Optimization of culture conditions and culture solution 
compositions for $\gamma$-aminobutyric acid accumulation in germinating mung bean (Vigna radiata L.). Journal of Biobased Materials \& Bioenergy, 12, 115-121.

Chen, L., Zhou, Y., He, Z., Liu, Q., Lai, S. \& Yang, H. (2018). Effect of exogenous ATP on the postharvest properties and pectin degradation of mung bean sprouts (Vigna radiata). Food Chemistry, 251, 9-17.

Chen, L., Tan, J.T.G., Zhao, X., Yang, D. \& Yang, H. (2019a). Energy regulated enzyme and non-enzyme-based antioxidant properties of harvested organic mung bean sprouts (Vigna radiata). LWT-Food Science and Technology, 107, 228-235.

Chen, L., Wu, J., Li, Z., Liu, Q., Zhao, X. \& Yang, H. (2019b). Metabolomic analysis of energy regulated germination and sprouting of organic mung bean (Vigna radiata) using NMR spectroscopy. Food Chemistry, 286, 87-97.

Chuang, C.-Y., Shi, Y.-C., You, H.-P., Lo, Y.-H. \& Pan, T.-M. (2011). Antidepressant effect of GABA-rich Monascus-fermented product on forced swimming rat model. Journal of Agricultural and Food Chemistry, 59, 3027-3034.

Diana, M., Quílez, J. \& Rafecas, M. (2014). Gamma-aminobutyric acid as a bioactive compound in foods: a review. Journal of Functional Foods, 10, 407-420.

Ding, J.Z., Yang, T.W., Feng, H. et al. (2016). Enhancing contents of $\gamma$-aminobutyric acid (GABA) and other micronutrients in dehulled rice during germination under normoxic and hypoxic conditions. Journal of Agricultural and Food Chemistry, 64, 1094-1102.

Fukumori, T., Kanemoto, S., Mizuno, H., Wakabayashi, K., Liu, H.Q. \& Ochiai, S. (2013). Grain or legume having increased content of functional component and a manufacturing method thereof. US8399037B2.

Ganesan, K. \& Xu, B.J. (2018). A critical review on phytochemical profile and health promoting effects of mung bean (Vigna radiata). Food Science and Human Wellness, 7, 11-33.

Guo, Y.X., Yang, R.Q., Chen, H., Song, Y. \& Gu, Z.X. (2012). Accumulation of $\gamma$-aminobutyric acid in germinated soybean (Glycine $\max$ L.) in relation to glutamate decarboxylase and diamine oxidase activity induced by additives under hypoxia. European Food Research and Technology, 234, 679-687.

Guo, H.M., Yang, X.S., Zhou, H.T. et al. (2017). Comparison of nutritional composition, aroma compounds, and biological activities of two kinds of tartary buckwheat tea. Journal of Food Science, 82, 1735-1741.

Huang, H.-Y., Hsu, T. \& Lin, B.-F. (2019). Gamma-aminobutyric acid decrease macrophages infiltration and suppresses inflammatory responses in renal injury. Journal of Functional Foods, 60, 110 .

Khwanchai, P., Chinprahast, N., Pichyangkura, R. \& Chaiwanichsiri, S. (2014). Gamma-aminobutyric acid and glutamic acid contents, and the GAD activity in germinated brown rice (Oryza sativa L.): effect of rice cultivars. Food Science and Biotechnology, 23, 373-379.

Kim, H.S., Lee, E.J., Lim, S.-T. \& Han, J.-A. (2015). Self-enhancement of GABA in rice bran using various stress treatments. Food Chemistry, 172, 657-662.

Komatsuzaki, N., Tsukahara, K., Toyoshima, H., Suzuki, T., Shimizu, N. \& Kimura, T. (2007). Effect of soaking and gaseous treatment on GABA content in germinated brown rice. Journal of Food Engineering, 78, 556-560.

Leventhal, A.G., Wang, Y.C., Pu, M.L., Zhou, Y.F. \& Ma, Y.Y. (2003). GABA and its agonists improved visual cortical function in senescent monkeys. Science, 300, 812-815.

Liao, W.-C., Wang, C.-Y., Shyu, Y.-T., Yu, R.-C. \& Ho, K.-C. (2013). Influence of preprocessing methods and fermentation of adzuki beans on $\gamma$-aminobutyric acid (GABA) accumulation by lactic acid bacteria. Journal of Functional Foods, 5, 1108-1115.

Locy, R.D., Wu, S.-J., Bisnette, J. et al. (2000). The regulation of GABA accumulation by heat stress in Arabidopsis. In: Plant
Tolerance to Abiotic Stresses in Agriculture: Role of Genetic Engineering. Pp. 39-52. Dordrecht: Springer.

Mayer, R.R., Cherry, J.H. \& Rhodes, D. (1990). Effects of heat shock on amino acid metabolism of cowpea cells. Plant Physiology, 94, 796-810.

Morrison, M.J., Frégeau-Reid, J.A. \& Cober, E.R. (2013). Seed protein, soaking duration, and soaking temperature effects on gamma aminobutyric acid concentration in short-season soybean. Crop Science, 53, 2563-2568.

Nikmaram, N., Dar, B.N., Roohinejad, S. et al. (2017). Recent advances in $\gamma$-amino butyric acid (GABA) properties in pulses: an overview. Journal of the Science of Food and Agriculture, 97, 26812689.

Nishimura, M., Yoshida, S.-I., Haramoto, M. et al. (2016). Effects of white rice containing enrichment gamma-aminobutyric acid on blood pressure. Journal of Traditional and Complementary Medicine, 6, 66-71.

Nonogaki, H., Bassel, G.W. \& Bewley, J.D. (2010). Germination-still a mystery. Plant Science, 179, 574-581.

Podlešáková, K., Ugena, L., Spíchal, L., Doležal, K. \& Diego, N.D. (2019). Phytohormones and polyamines regulate plant stress responses by altering GABA pathway. New Biotechnology, 48, 5365.

Qin, P.Y., Song, W.W., Yang, X.S. et al. (2014). Regional distribution of protein and oil compositions of soybean cultivars in China. Crop Science, 54, 1139-1146.

Roohinejad, S., Omidizadeh, A., Mirhosseini, H. et al. (2009). Effect of hypocholesterolemic properties of brown rice varieties containing different gamma aminobutyric acid (GABA) levels on SpragueDawley male rats. Journal of Food Agriculture and Environment, 7, 197-203.

Roohinejad, S., Omidizadeh, A., Mirhosseini, H. et al. (2011). Effect of pre-germination time on amino acid profile and gamma amino butyric acid (GABA) contents in different varieties of Malaysian brown rice. International Journal of Food Properties, 14, 13861399.

Sharma, S., Saxena, D.C. \& Riar, C.S. (2018). Changes in the GABA and polyphenols contents of foxtail millet on germination and their relationship with in vitro antioxidant activity. Food Chemistry, 245, 863-870.

Shi, Z.X., Yao, Y., Zhu, Y.Y. \& Ren, G.X. (2016). Nutritional composition and antioxidant activity of twenty mung bean cultivars in China. The Crop Journal, 4, 398-406.

Signorelli, S., Dans, P.D., Coitiño, E.L., Borsani, O. \& Monza, J. (2015). Connecting proline and $\gamma$-aminobutyric acid in stressed plants through non-enzymatic reactions. PLoS One, 10, 1-14.

Tiansawang, K., Luangpituksa, P., Varanyanond, W. \& Hansawasdi, C. (2016). GABA ( $\gamma$-aminobutyric acid) production, antioxidant activity in some germinated dietary seeds and the effect of cooking on their GABA content. Food Science and Technology, 36, 313321 .

Wakte, K.V., Kad, T.D., Zanan, R.L. \& Nadaf, A.B. (2011). Mechanism of 2-acetyl-1-pyrroline biosynthesis in Bassia latifolia Roxb. flowers. Physiology and Molecular Biology of Plants, 17, 231-237.

Xing, S.G., Jun, Y.B., Hau, Z.W. \& Liang, L.Y. (2007). Higher accumulation of $\gamma$-aminobutyric acid induced by salt stress through stimulating the activity of diamine oxidases in Glycine max (L.) Merr. roots. Plant Physiology and Biochemistry, 45, 560-566.

Xu, J.G. \& Hu, Q.P. (2014). Changes in $\gamma$-aminobutyric acid content and related enzyme activities in Jindou 25 soybean (Glycine max L.) seeds during germination. LWT-Food Science and Technology, 55, 341-346.

Yang, R.Q., Guo, Q.H. \& Gu, Z.X. (2013). GABA shunt and polyamine degradation pathway on $\gamma$-aminobutyric acid accumulation in germinating fava bean (Vicia faba L.) under hypoxia. Food Chemistry, 136, 152-159. 
Youn, Y.-S., Park, J.-K., Jang, H.-D. \& Rhee, Y.-W. (2011). Sequential hydration with anaerobic and heat treatment increases GABA ( $\gamma$-aminobutyric acid) content in wheat. Food Chemistry, 129, 1631-1635.

Zhao, G.-C., Xie, M.-X., Wang, Y.-C. \& Li, J.-Y. (2017). Molecular mechanisms underlying $\gamma$-aminobutyric acid (GABA) accumulation in giant embryo rice seeds. Journal of Agricultural and Food Chemistry, 65, 4883-4889.

\section{Supporting Information}

Additional Supporting Information may be found in the online version of this article:
Figure S1. Thirty-four Chinese mung bean varieties pictures.

Figure S2. The GABA content of native and $\mathrm{HRH}-$ treated mung beans in different provinces.

Figure S3. Representative FAA chromatogram patterns of native and $\mathrm{HRH}$-treated mung bean. 